\title{
Prognostic value of LINE-1 methylation level in 321 patients with primary liver cancer including hepatocellular carcinoma and intrahepatic cholangiocarcinoma
}

Tatsunori Miyata ${ }^{1}$, Yo-Ichi Yamashita ${ }^{1}$, Yoshifumi Baba ${ }^{1}$, Kazuto Harada ${ }^{1}$, Takanobu Yamao', Naoki Umezaki ${ }^{1}$, Masayo Tsukamoto ${ }^{1}$, Yuki Kitano, Kensuke Yamamura $^{1}$, Kota Arima ${ }^{1}$, Shigeki Nakagawa ${ }^{1}$, Hirohisa Okabe ${ }^{1}$, Katsunori Imai ${ }^{1}$, Daisuke Hashimoto ${ }^{1}$, Akira Chikamoto ${ }^{1}$, Mototsugu Shimokawa² and Hideo Baba ${ }^{1}$

${ }^{1}$ Department of Gastroenterological Surgery, Graduate School of Life Sciences, Kumamoto University, Kumamoto, Japan

${ }^{2}$ Clinical Research Institute, National Kyushu Cancer Center, Fukuoka, Japan

Correspondence to: Hideo Baba, email: hdobaba@kumamoto-u.ac.jp

Keywords: LINE-1; methylation; epigenetics; primary liver cancer; prognosis

Received: October 18, $2017 \quad$ Accepted: March 22, $2018 \quad$ Published: April 17, 2018

Copyright: Miyata et al. This is an open-access article distributed under the terms of the Creative Commons Attribution License 3.0 (CC BY 3.0), which permits unrestricted use, distribution, and reproduction in any medium, provided the original author and source are credited.

\section{ABSTRACT}

Background: The methylation level of long interspersed nucleotide element-1 (LINE-1) is a good surrogate marker of the global DNA methylation level. The relationship between LINE-1 methylation level and prognosis in primary liver cancer (PLC) patients remains unclear.

Results: LINE-1 methylation levels were significantly lower in HCC and CHCC-CC tissues, but not in ICC tissues, than those in noncancerous liver parenchyma (HCC: $p<0.0001$; CHCC-CC: $p<0.001$; and ICC: $p=0.053)$. HCC cases with hypomethylated LINE-1 had significantly shorter relapse-free survival (RFS) $(\log -r a n k, p=0.008)$; however, this was not observed for the CHCC-CC or ICC cases. Multivariate Cox regression analysis revealed a significantly higher $\mathrm{HCC}$ recurrence rate in the group with hypomethylated LINE-1 (hazard ratio, 1.62; 95\% confidence interval, 1.06-2.58; $p=0.025$ ).

Conclusions: The genome-wide DNA hypomethylation status estimated via LINE-1 methylation levels might be indicative of poor RFS in patients with HCC but not ICC or cHCC-CC.

Methods: We evaluated the level of LINE-1 methylation in 321 cases of curatively resected PLC $\{231$ hepatocellular carcinoma (HCC), 19 combined hepatocellular and cholangiocarcinoma (CHCC-CC) and 71 intrahepatic cholangiocarcinoma (ICC)\} via pyrosequencing of formalin-fixed paraffin-embedded (FFPE) tissues and examined its prognostic value.

\section{INTRODUCTION}

Primary liver cancer (PLC) is the sixth most common cancer and the second leading cause of cancer death worldwide [1]. Pathologically, approximately $85-90 \%$ of PLC can be classified as hepatocellular carcinoma (HCC) and $5-10 \%$ as intrahepatic cholangiocarcinoma (ICC), with combined hepatocellular and cholangiocarcinoma (cHCC$\mathrm{CC}$ ) representing a small portion of PLC [2-4]. Many cases of HCC arise from cirrhosis resulting from chronic infection by hepatitis $\mathrm{B}$ and $\mathrm{C}$ virus (HBV and $\mathrm{HCV}$, respectively), alcoholic injury, and non-alcoholic fatty liver disease (NAFLD), which are increasing in incidence with changes in lifestyles [5, 6]. ICC is an aggressive cancer arising from epithelial cells of the bile duct or hepatocytes [7], and its development has been associated with primary sclerosing cholangitis, hepatitis virus infection, alcohol consumption, smoking, fatty liver disease, diabetes, cholelithiasis, and choledocholithiasis. In addition, risk factors of ICC vary depending on the region [8]. $\mathrm{cHCC}-\mathrm{CC}$ is currently 
defined as an unequivocal mixture of both HCC and ICC. According to the recent definition from the WHO, the cHCC-CC category comprises two histological forms: a classic type and a subtype with stem cell-like features [9]. All three cancers belong to the same category as PLC; however, clinically, ICC and cHCC-CC often show much more aggressive behavior with poorer prognosis than does HCC, with no standard treatment other than curative surgical resection [2-4]. Thus, the clinical features of these three types of cancer are likely distinct.

Cancer initiation and progression are caused by concurrent changes in multiple genes via genetic and epigenetic alterations leading to the activation of oncogenes or the suppression of tumor suppressor genes [10]. Along with genetic mutations, epigenetic changes such as DNA methylation and histone acetylation are important for carcinogenesis and tumor development $[11,12]$. Cancer cells exhibit two types of alterations in DNA methylation: one is global DNA hypomethylation, and the other is site-specific $\mathrm{CpG}$ island promoter hypermethylation $[13,14]$. Global DNA hypomethylation plays an important role in genomic instability, and site-specific promoter hypermethylation can silence tumor suppressor genes, leading to cancer development [15-18]. Since the long interspersed nucleotide element-1 (LINE-1) retrotransposon constitutes a substantial portion of the human genome (approximately 17\%), the methylation status of LINE-1 reflects the global DNA methylation status [19]. LINE1 hypomethylation is associated with poor prognosis in esophageal, gastric, colorectal, pancreas and breast cancer [20-24], and it can be measured in a cost-effective manner via high-throughput pyrosequencing techniques [25-27]. Therefore, the methylation level of LINE-1 may be an effective biomarker for prognostic prediction.

We previously reported that LINE-1 hypomethylation was associated with poor prognosis in 208 patients with HCC [28]; However, there are no reports that comprehensively analyzed LINE-1 methylation levels in PLCs including ICC and cHCC-CC. Therefore, the aims of this study are as follows: to confirm the association between LINE-1 methylation levels and prognosis in HCC by using a greater number of samples; to examine prognostic significance in ICC and $\mathrm{CHCC}-\mathrm{CC}$; to analyze the difference in the characteristics of LINE-1 methylation levels among the various subtypes of PLC (i.e., HCC, ICC, cHCC-CC).

\section{RESULTS}

\section{LINE-1 methylation level in PLC}

We examined LINE-1 methylation levels in 321 PLC patients including $231 \mathrm{HCC}, 19 \mathrm{cHCC}-\mathrm{CC}$ and $71 \mathrm{ICC}$ cases. LINE-1 methylation levels in cancerous tissues were significantly different among the three subtypes of PLC (HCC vs cHCC-CC; $p<0.001$, HCC vs ICC; $p<0.0001)$. HCC cases had the lowest LINE-1 methylation levels among all PLC subtypes (mean: 65.7\%, range: 21.5-99.1\%). However, LINE-1 methylation levels were not significantly different between cHCC-CC (mean: 76.4\%, range: 54.0-88.0\%) and ICC (mean: 81.6\%, range: 47.0-91.0\%) $(p=0.075)$ (Figure 1). For HCC, LINE-1 methylation levels were lower in the HCV-infected groups than those in the non-HCVinfected group, while similar differences were not observed for cHCC-CC or ICC (Table 1).

\section{LINE-1 methylation level in PLC and noncancerous liver tissue}

Next, we examined LINE-1 methylation levels in 201 PLC tissues and their matched noncancerous liver parenchyma, including $111 \mathrm{HCC}, 19 \mathrm{cHCC}-\mathrm{CC}$ and 71 ICC cases. LINE-1 methylation levels in the PLC tissues (vs. noncancerous liver parenchyma) were distributed as follows: mean 69.8 (80.6); median 72.0 (82.0); standard deviation (SD) 14.7 (10.9); and range 6-99.1 (9.3-97.3). LINE-1 methylation levels were significantly lower in PLC tissues than those in noncancerous liver parenchyma ( $p<0.0001)$ (Figure 2A). In addition, LINE1 methylation levels were significantly lower in $\mathrm{HCC}$ and $\mathrm{cHCC}-\mathrm{CC}$ than those in their matched parenchyma (HCC: $p<0.0001$, cHCC-CC: $p<0.001$ ). In Student's $t$ test, LINE-1 methylation level was also lower in ICC; however, this difference was not statistically significant $(p=0.053)$ (Figure 2B). Similarly, in paired $t$ test, there were significantly differences between cancerous tissue and noncancerous tissue in $\mathrm{HCC}(p<0.0001)$ and cHCC-CC $(p<0.001)$, however, there was not in ICC $(p=0.167)$. These differences were also observed between PLC and noncancerous liver parenchyma irrespective of the presence or absence of hepatitis virus infection (Supplementary Figure 1). However, there was no effect of hepatitis virus infection on LINE-1 methylation level in non-cancerous part of PLC (HCC; $\mathrm{p}=0.604$, cHCC-CC; $p=0.373$ and ICC; $p=0.876$ ) (Supplementary Figure 1). Therefore, the LINE-1 methylation status between cancer and noncancerous liver parenchyma was different for $\mathrm{HCC}$ and cHCC-CC but not for ICC.

\section{Association between LINE-1 methylation level and clinical, epidemiological, and pathological variables}

We next examined the relationship between LINE-1 methylation level in PLC and various clinical or pathological variables. There were significant correlations between LINE-1 methylation level and HCV-specific antibody ( $\mathrm{HCV} \mathrm{Ab})$-positivity $(p=0.039)$, des-gammacarboxy prothrombin (DCP) $(p=0.032)$ and $\mathrm{F}$ stage $(p=0.024)$ in HCC and between LINE-1 methylation level and tumor number in $\mathrm{cHCC}-\mathrm{CC}(p=0.038)$. The 
other factors were not significantly different between the groups in any of the subtypes of PLC (Table 1).

\section{LINE-1 methylation level and patient prognosis}

A follow-up study on the 321 patients revealed 188 cancer recurrences $(58.6 \%)$ and 117 deaths (36.4\%). The median follow-up time for the diseased patients was 4.0 years. Using LINE-1 methylation level as a quartile categorical variable for each subtype of PLC. Using the methylation level as a quartile categorical variable (i.e. first quartile cases $[Q 1 ; \geq 75.28 \%$, second quartile cases [Q2; 65.61-75.28\%], third quartile cases [Q3; 56.49-65.61\%], and fourth quartile cases [Q4; < $56.49 \%$ ] in HCC; [Q1; $\geq 84.0 \%$ ], [Q2; 78.0-84.0\%], [Q3; 72.0-78.0\%] and Q4; < 72.0\% in cHCC-CC; [Q1; $\geq 88.0 \%$ ], [Q2; 84.0-88.0\%], [Q3; 80.0-84.0\%] and [Q4; $<80.0 \%$ ] in ICC). We adopted a dichotomous LINE-1 methylation level, defining cases in Q1 as the 'LINE-1 hypermethylation group' and combining cases in Q2 to Q4 into the 'LINE-1 hypomethylation group' for each subtype of PLC (Supplementary Table 1). Then, we analyzed the correlation between the LINE-1 methylation status and prognosis for the 321 PLC patients. Interestingly, LINE1 hypomethylation was associated with unfavorable RFS for HCC ( $p=0.008)$; however, there were no significant differences between the LINE-1 hypomethylation and hypermethylation groups for $\mathrm{cHCC}-\mathrm{CC}(p=0.067)$ or ICC $(p=0.357)$ (Figure 3$)$. Multivariate Cox regression analysis also revealed that LINE-1 hypomethylation was an independent prognostic factor for HCC (HR; 1.62, 95\% CI; 1.06-2.58, $p=0.025$ ) (Table 2). Additionally, we examined whether the effect of LINE-1 hypomethylation on cancer recurrence was modified by any of the clinical and pathological variables, including age, sex, hepatitis virus infection (HBV or HCV), Child-Pugh classification, ICG-R15, stage of fibrosis, tumor size, number of tumors, and differentiation. The relationship between LINE1 methylation level and RFS rate was significantly modified by hepatitis virus infection ( $p$ of interaction $=0.008$ ) (Figure 4). On the other hand, in univariate and multivariate Cox regression analyses for ICC, LINE-1 hypomethylation was not an independent prognostic factor $(p=0.372)$, while vascular invasion $(p=0.007)$ and lymph node metastasis $(p=0.019)$ were independent prognostic factors (Table 2). We did not analyze the prognostic factors for cHCC-CC in RFS and OS because of the small number of cases.

Finally, we analyzed the relationship between OS and LINE-1 methylation level. There were no significant differences between the hyper- and hypomethylation groups in any subtype of PLC (Supplementary Figure 2). Thus, LINE-1 hypomethylation was associated with poor RFS in HCC patients only.

\section{DISCUSSION}

To the best of our knowledge, this is the first study examining the prognostic value of LINE-1 methylation levels in PLC including $\mathrm{HCC}$, ICC and $\mathrm{cHCC}-\mathrm{CC}$. Our results using 321 PLC FFPE samples showed that LINE-1 methylation levels were lower in $\mathrm{HCC}$ and $\mathrm{cHCC}-\mathrm{CC}$ tissues but not in ICC tissues than those in the matched noncancerous liver parenchyma. This suggests that there

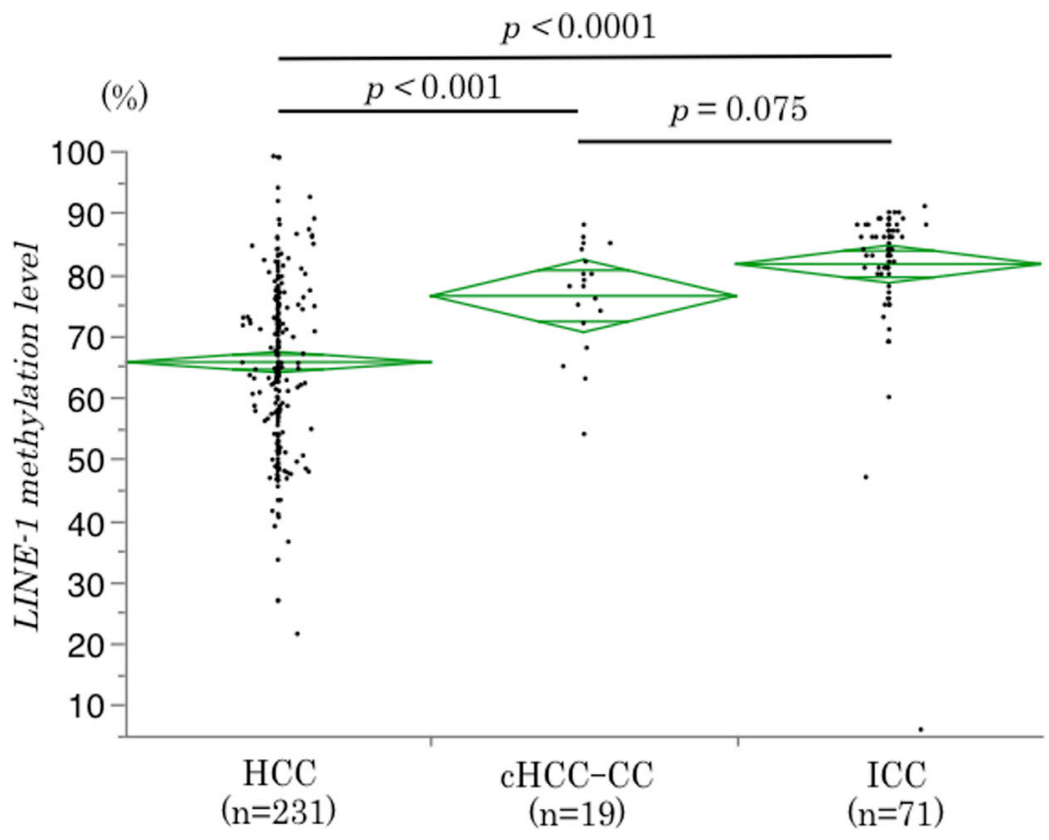

Figure 1: Differences in LINE-1 methylation levels among PLC subtypes. LINE-1 methylation levels were different among PLC subtypes, with the lowest levels for HCC. The diamonds mean 95\% confidence interval of the mean value. 
Table 1: Association between the LINE-1 methylation level in cancerous tissues and clinicopathological characteristics of 321 patients with primary liver cancer

\begin{tabular}{|c|c|c|c|c|c|c|c|c|c|}
\hline Variables & $\begin{array}{c}\text { HCC } \\
(n=231)\end{array}$ & $\begin{array}{c}\text { LINE-1 } \\
\text { methylation } \\
\text { level }(\%) \\
{[\text { mean } \pm \text { SE] }}\end{array}$ & $P$ & $\begin{array}{c}\text { cHCC- } \\
\text { CC } \\
(n=19)\end{array}$ & $\begin{array}{c}\text { LINE-1 } \\
\text { methylation } \\
\text { level }(\%) \\
{[\text { mean } \pm \text { SE] }}\end{array}$ & $P$ & $\begin{array}{c}\text { ICC } \\
(n=71)\end{array}$ & $\begin{array}{c}\text { LINE-1 methylation } \\
\text { level }(\%) \\
{[\text { mean } \pm \text { SE] }}\end{array}$ & $P$ \\
\hline Age (yrs) & & & 0.489 & & & 0.361 & & & 0.991 \\
\hline$\geq 75$ & 39 & $64.3 \pm 2.2$ & & 2 & $75.8 \pm 2.2$ & & 16 & $81.6 \pm 2.9$ & \\
\hline$<75$ & 192 & $65.9 \pm 1.0$ & & 17 & $82.0 \pm 6.3$ & & 55 & $81.6 \pm 1.6$ & \\
\hline Sex & & & 0.869 & & & 0.343 & & & 0.913 \\
\hline Male & 189 & $65.6 \pm 1.0$ & & 16 & $75.6 \pm 2.2$ & & 22 & $81.8 \pm 2.5$ & \\
\hline Female & 42 & $66.0 \pm 2.1$ & & 3 & $81.0 \pm 5.1$ & & 49 & $81.5 \pm 1.7$ & \\
\hline HBs Ag & & & 0.117 & & & 0.615 & & & 0.981 \\
\hline Positive & 66 & $67.9 \pm 1.7$ & & 5 & $78.2 \pm 4.0$ & & 8 & $81.5 \pm 4.1$ & \\
\hline Negative & 164 & $64.7 \pm 1.1$ & & 14 & $75.8 \pm 2.4$ & & 63 & $81.6 \pm 1.5$ & \\
\hline $\mathrm{HCV} A b$ & & & 0.039 & & & 0.228 & & & 0.276 \\
\hline Positive & 113 & $63.7 \pm 1.3$ & & 7 & $73.1 \pm 3.3$ & & 14 & $84.6 \pm 3.1$ & \\
\hline Negative & 117 & $67.5 \pm 1.3$ & & 12 & $78.3 \pm 2.5$ & & 57 & $80.8 \pm 1.5$ & \\
\hline $\begin{array}{l}\text { Child-Pugh } \\
\text { classification }\end{array}$ & & & 0.269 & & & - & & & 0.590 \\
\hline A & 206 & $66.0 \pm 1.0$ & & 19 & $76.4 \pm 2.0$ & & 69 & $81.5 \pm 1.4$ & \\
\hline B & 25 & $62.8 \pm 2.7$ & & 0 & & & 2 & $86.0 \pm 8.3$ & \\
\hline ICG-R15 & & & 0.196 & & & 0.472 & & & 0.445 \\
\hline$\geq 10$ & 91 & $64.4 \pm 1.5$ & & 8 & $78.1 \pm 3.3$ & & 13 & $83.7 \pm 3.4$ & \\
\hline$<10$ & 114 & $66.9 \pm 1.3$ & & 10 & $74.9 \pm 2.9$ & & 51 & $80.8 \pm 1.7$ & \\
\hline $\begin{array}{l}\text { Total bilirubin } \\
(\mathrm{mg} / \mathrm{dl})\end{array}$ & & & 0.729 & & & 0.450 & & & 0.686 \\
\hline$>1.0$ & 43 & $66.3 \pm 2.1$ & & 4 & $79.5 \pm 4.8$ & & 10 & $80.2 \pm 3.7$ & \\
\hline$\leq 1.0$ & 188 & $65.5 \pm 1.0$ & & 15 & $75.6 \pm 2.3$ & & 61 & $81.8 \pm 1.5$ & \\
\hline Albumin (g/dl) & & & 0.801 & & & 0.853 & & & 0.072 \\
\hline$>3.9$ & 143 & $65.5 \pm 1.1$ & & 13 & $76.2 \pm 2.5$ & & 46 & $76.8 \pm 1.7$ & \\
\hline$\leq 3.9$ & 88 & $66.0 \pm 1.5$ & & 6 & $77.0 \pm 3.7$ & & 25 & $85.0 \pm 2.9$ & \\
\hline PT (\%) & & & 0.929 & & & 0.439 & & & 0.258 \\
\hline$\geq 85$ & 175 & $65.7 \pm 1.0$ & & 16 & $77.1 \pm 2.2$ & & 63 & $81.0 \pm 1.5$ & \\
\hline$<85$ & 56 & $65.5 \pm 1.8$ & & 3 & $72.7 \pm 5.2$ & & 8 & $86.0 \pm 4.1$ & \\
\hline $\operatorname{AFP}(\mathrm{ng} / \mathrm{mL})$ & & & 0.488 & & & - & & & - \\
\hline$>7.0$ & 154 & $65.2 \pm 1.1$ & & - & - & & - & - & \\
\hline$\leq 7.0$ & 77 & $66.6 \pm 1.6$ & & - & - & & - & - & \\
\hline $\mathrm{DCP}(\mathrm{mAU} / \mathrm{mL})$ & & & 0.032 & & & - & & & - \\
\hline$\geq 40$ & 164 & $64.4 \pm 1.1$ & & - & - & & - & - & \\
\hline$<40$ & 67 & $68.7 \pm 1.7$ & & - & - & & - & - & \\
\hline CA19-9 (U/mL) & & & - & & & - & & & 0.777 \\
\hline$\geq 37$ & - & - & & - & - & & 22 & $82.2 \pm 2.5$ & \\
\hline$<37$ & - & - & & - & - & & 49 & $81.3 \pm 1.7$ & \\
\hline Differenciation & & & 0.358 & & & - & & & 0.965 \\
\hline Well-moderate & 176 & $66.1 \pm 1.0$ & & - & - & & 54 & $81.4 \pm 1.6$ & \\
\hline Poor & 53 & $64.1 \pm 1.9$ & & - & - & & 11 & $81.5 \pm 3.6$ & \\
\hline
\end{tabular}




\begin{tabular}{|c|c|c|c|c|c|c|c|c|c|}
\hline$\geq 50$ & 82 & $66.5 \pm 1.5$ & & 8 & $76.5 \pm 3.2$ & & 22 & $82.2 \pm 2.5$ & \\
\hline$<50$ & 149 & $65.2 \pm 1.1$ & & 11 & $76.4 \pm 2.7$ & & 49 & $81.3 \pm 1.7$ & \\
\hline Tumor number & & & 0.299 & & & 0.038 & & & 0.893 \\
\hline Single & 165 & $65.1 \pm 1.1$ & & 13 & $79.2 \pm 2.2$ & & 57 & $81.7 \pm 1.6$ & \\
\hline Multiple & 66 & $67.1 \pm 1.7$ & & 6 & $70.3 \pm 3.3$ & & 14 & $81.2 \pm 3.1$ & \\
\hline Vascular invasion & & & 0.565 & & & 0.809 & & & 0.239 \\
\hline Present & 106 & $65.1 \pm 1.3$ & & 13 & $76.8 \pm 2.5$ & & 34 & $83.3 \pm 2.0$ & \\
\hline Absent & 125 & $66.1 \pm 1.2$ & & 6 & $75.7 \pm 3.7$ & & 37 & $80.0 \pm 1.9$ & \\
\hline LN status & & & - & & & - & & & 0.276 \\
\hline 0 & - & - & & - & - & & 22 & $83.6 \pm 2.5$ & \\
\hline 1 & - & - & & - & - & & 9 & $76.2 \pm 3.9$ & \\
\hline $\mathrm{X}$ & - & - & & - & - & & 40 & $81.7 \pm 1.8$ & \\
\hline F stage & & & 0.024 & & & - & & & 0.343 \\
\hline F1-3 & 140 & $67.0 \pm 1.1$ & & - & - & & 60 & $80.9 \pm 1.5$ & \\
\hline F4 & 80 & $62.7 \pm 1.5$ & & - & - & & 7 & $85.4 \pm 4.5$ & \\
\hline Procedure & & & 0.178 & & & 0.607 & & & 0.209 \\
\hline Minor & 172 & $65.0 \pm 1.0$ & & 12 & $77.3 \pm 2.6$ & & 26 & $83.9 \pm 2.7$ & \\
\hline Major & 59 & $67.7 \pm 1.8$ & & 7 & $75.0 \pm 3.4$ & & 45 & $80.3 \pm 1.7$ & \\
\hline
\end{tabular}

Abbreviations: HCC, Hepatocellular carcinoma; ICC, Intrahepatic cholangiocarcinoma; cHCC-CC, Combined hepatocellular and cholangiocarcinoma; HBs Ag, Hepatitis B surface antigen; HCV Ab, Hepatitis C virus-specific antibody; ICG-R15, Indocyanine green retention rate at 15 minutes; PT, Prothrombin time; AFP, Alpha-fetoprotein; DCP, Des-gamma-carboxy prothrombin; CA19-9: Carbohydrate antigen 19-9; LN, Lymph node.

may be differences in the epigenetic status among the subtypes of PLC. In addition, LINE-1 hypomethylation was associated with unfavorable clinical outcomes in patients with HCC only. Thus, the current study demonstrated differences in epigenetic status among PLC subtypes and the association between LINE-1 methylation and prognosis in PLC patients.

Our results suggested that global DNA hypomethylation, which leads to chromosome instability and fragility, is correlated with cancer initiation and progression in HCC and cHCC-CC but not in ICC. Udali et al. reported significantly lower levels of DNA methylation in HCC than those in cholangiocarcinoma (CC) tissues and comparable levels between $\mathrm{CC}$ and matched noncancerous liver parenchyma and gall bladder tissues [29]. Our data are consistent with their results. Thus, although HCC and ICC are similar subtypes of PLC, their epigenetic status may be distinct. One of the reasons behind the differences may be the presence of isocitrate dehydrogenase 1 (IDH1) and IDH2 mutations in ICC. IDH1 and 2 are enzymes that catalyze the oxidative decarboxylation of isocitrate to $\alpha$-ketoglutarate and known as one of the driver genes of ICC [30]. IDH1/2 mutations are observed in 15 to $20 \%$ of ICC cases [31, 32] and result in the production of the 2-hydroxyglutarate (2$\mathrm{HG}$ ), which inhibits the , 2-HG contributes to maintaining (TET) proteins [33]. TET family enzymes (TET1, TET2, and TET3) are implicated in DNA demethylation through their dioxygenase activity that converts 5-methylcytosine to 5-hydroxymethylcytosine [34]. Thus, 2-HG contributes to maintaining DNA hypermethylation by inhibiting TET2 activity. Therefore, IDH1/2 mutations lead to DNA hypermethylation in ICC, leading LINE-1 hypermethylation. However, IDH $1 / 2$ mutations only partly account for the differences in the methylation patterns in ICC [31, 32], and there may be other mechanisms underlying the hypermethylation of DNA in ICC compared to that in the other PLC subtypes. In addition, there was only one patient with neoadjuvant chemotherapy in ICC using FOLFOX (leucovorin and fluorouracil and oxaliplatin): the presence of preoperative chemotherapy would affect the LINE-1 methylation level in cancerous or noncancerous tissues of ICC; however, we cannot refer this issue because of the lack of samples to evaluate. Interestingly, LINE-1 methylation levels were significantly lower in $\mathrm{cHCC}-\mathrm{CC}$ cancerous tissues than those in noncancerous liver parenchyma, suggesting that $\mathrm{cHCC}-\mathrm{CC}$ was more similar to $\mathrm{HCC}$ than to ICC in terms of epigenetic status.

LINE-1 hypomethylation is associated with poor prognosis in several cancers [20-24]. The underlying mechanisms may be as follows: First, global DNA hypomethylation contributes to cancer development by inducing genomic instability [15]. Second, hypomethylation at gene regulatory regions or loss of genetic imprinting promotes the expression 
of oncogenes [35]. Third, LINE-1 hypomethylation activates oncogenes (e.g., c-MET [36]) and cell cyclerelated genes (e.g., CDK6 [28]), which are associated with cancer progression [37]. Therefore, LINE-1 hypomethylation may also be associated with cancer progression. However, we found no association between LINE-1 hypomethylation and OS in any of the three subtypes of PLC. The present study showed that LINE-1 hypomethylation is related to short RFS in HCC patients only. We can explain this discrepancy as follows: First, re-resection for recurrent $\mathrm{HCC}$ can prolong OS [38, 39]. Therefore, although the RFS for HCC was short in the LINE-1 hypomethylation group, OS was not. Second, the sample size for cHCC-CC was very small. However, we could observe a trend toward poorer RFS and OS in the LINE-1 hypomethylation group than those in the LINE-

A
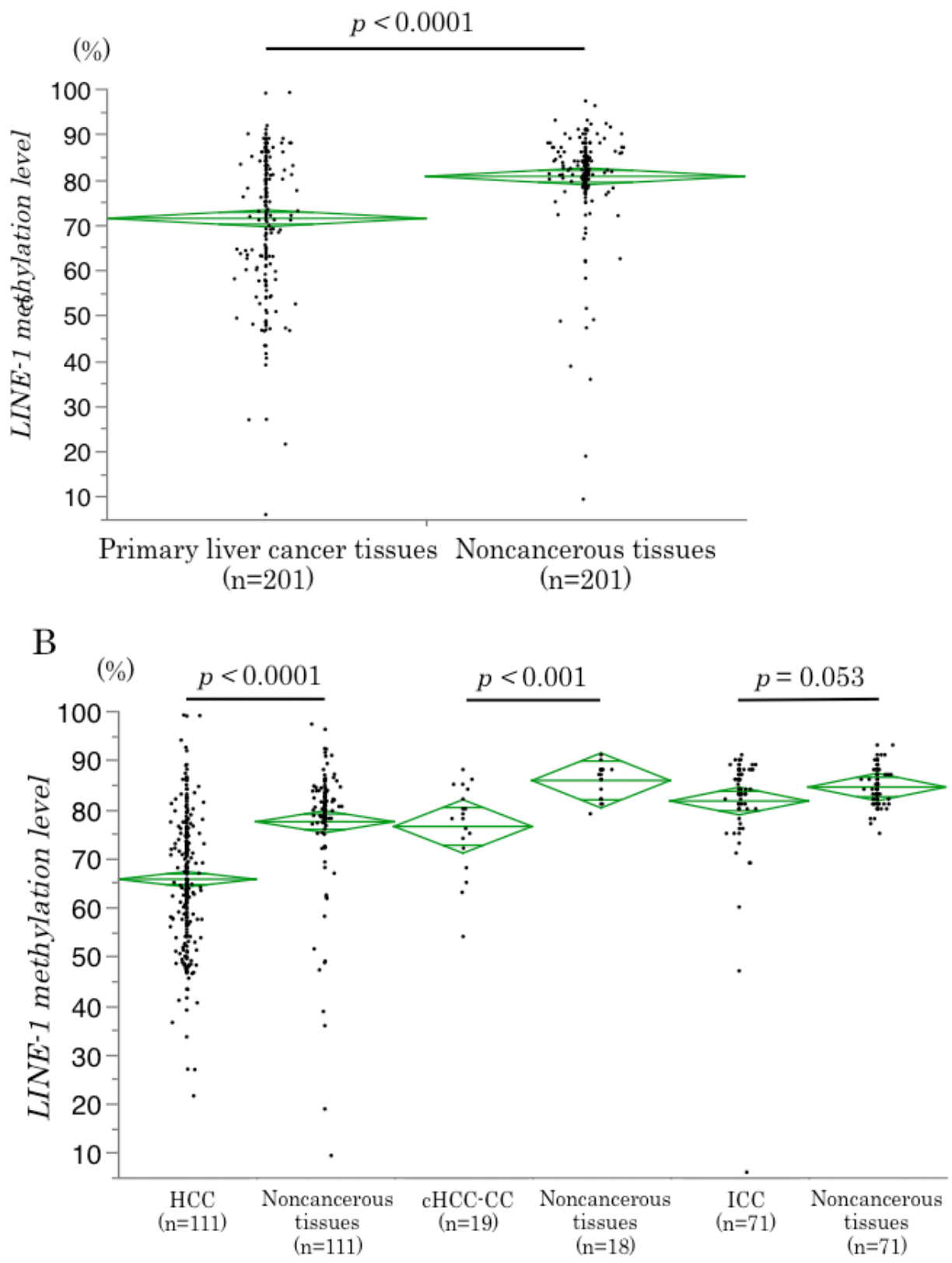

Figure 2: LINE-1 methylation levels in cancerous and noncancerous tissues. (A) LINE-1 methylation levels in cancerous tissues and noncancerous liver parenchyma in 201 PLC patients. The levels were significantly lower in the cancerous tissues than in the noncancerous liver tissues $(p<0.0001)$. (B) LINE-1 methylation levels in cancerous tissues and noncancerous liver tissues for each histological subtype of PLC. The levels were significantly lower in the cancerous tissues than in the noncancerous liver parenchyma for HCC $(p<0.0001)$ and cHCC-CC $(p<0.001)$, but not for ICC $(p=0.053)$. The diamonds mean 95\% confidence interval of the mean value. 
Table 2: Univariate and multivariate analyses for RFS

\begin{tabular}{|c|c|c|c|c|c|c|c|c|}
\hline \multirow{3}{*}{ Variables } & \multicolumn{4}{|c|}{ HCC $(n=231)$} & \multicolumn{4}{|c|}{$\operatorname{ICC}(n=71)$} \\
\hline & \multicolumn{2}{|c|}{ Univariate } & \multicolumn{2}{|c|}{ Multivariate } & \multicolumn{2}{|c|}{ Univariate } & \multicolumn{2}{|c|}{ Multivariate } \\
\hline & $\begin{array}{c}\text { HR } \\
(95 \% \mathrm{CI})\end{array}$ & $P$ & $\begin{array}{c}\text { HR } \\
(95 \% \mathrm{CI})\end{array}$ & $\boldsymbol{P}$ & $\begin{array}{c}\text { HR } \\
(95 \% \mathrm{CI})\end{array}$ & $P$ & $\begin{array}{c}\text { HR } \\
(95 \% \mathrm{CI})\end{array}$ & $\boldsymbol{P}$ \\
\hline Age $\geq 75$ yrs vs. $<75$ yrs & $1.11(0.72-1.67)$ & 0.607 & & & $1.65(0.80-3.15)$ & 0.165 & & \\
\hline Male vs. Female & $1.33(0.89-2.10)$ & 0.171 & & & $2.01(1.09-3.59)$ & 0.026 & & \\
\hline $\begin{array}{l}\text { Hapatitis virus infection positive } \\
\text { vs.negative }\end{array}$ & $1.24(0.86-1.83)$ & 0.253 & & & $0.73(0.38-1.33)$ & 0.305 & & \\
\hline HBs Ag positive vs.negative & $0.92(0.64-1.32)$ & 0.674 & & & $0.95(0.36-2.06)$ & 0.897 & & \\
\hline $\mathrm{HCV}$ Ab positive vs.negative & $1.24(0.90-1.71)$ & 0.189 & & & $0.67(0.29-1.35)$ & 0.276 & & \\
\hline Child-Pugh classification B vs. A & $1.51(0.89-2.41)$ & 0.122 & & & $1.44(0.23-4.70)$ & 0.636 & & \\
\hline ICG-R $15 \geqq 10$ vs. $<10 \%$ & $1.65(1.17-2.33)$ & 0.005 & $\begin{array}{c}1.58(1.12- \\
2.25)\end{array}$ & 0.010 & $0.87(0.39-1.75)$ & 0.720 & & \\
\hline AFP $>7.0$ vs. $\leq 7.0 \mathrm{ng} / \mathrm{mL}$ & $1.21(0.86-1.72)$ & 0.285 & & & - & - & & \\
\hline $\mathrm{DCP} \geq 40$ vs. $<40 \mathrm{mAU} / \mathrm{mL}$ & $1.17(0.82-1.70)$ & 0.394 & & & - & - & & \\
\hline CA19-9 $\geq 37$ vs. $<37 \mathrm{U} / \mathrm{mL}$ & - & - & & & $1.71(0.95-3.03)$ & 0.075 & & \\
\hline F stage F4 vs. F1-3 & $1.34(0.96-1.88)$ & 0.087 & & & $0.88(0.30-2.04)$ & 0.789 & & \\
\hline $\begin{array}{l}\text { Poor vs. well-moderate } \\
\text { differentiation }\end{array}$ & $1.03(0.69-1.50)$ & 0.887 & & & $1.06(0.48-2.12)$ & 0.870 & & \\
\hline Tumor size $\geqq 50$ vs. $<50 \mathrm{~mm}$ & $1.33(0.89-2.10)$ & 0.208 & & & $2.03(1.09-3.66)$ & 0.026 & & \\
\hline Multiple tumors vs. single tumor & $1.24(0.89-1.72)$ & $<0.0001$ & $\begin{array}{c}2.25(1.56- \\
3.22)\end{array}$ & $<0.0001$ & $1.33(0.89-2.10)$ & 0.367 & & \\
\hline $\begin{array}{l}\text { Vascular invasion present vs. } \\
\text { absent }\end{array}$ & $1.34(0.97-1.84)$ & 0.077 & & & $2.15(1.22-3.85)$ & 0.009 & $\begin{array}{c}2.20(1.24- \\
3.96)\end{array}$ & 0.007 \\
\hline LN status pN1 vs. pN0, NX & - & - & & - & $2.56(1.15-5.11)$ & 0.023 & $\begin{array}{c}2.66(1.19- \\
5.31)\end{array}$ & 0.019 \\
\hline $\begin{array}{l}\text { LINE-1 hypomethylation vs. } \\
\text { hypermethylation }\end{array}$ & $1.73(1.17-2.67)$ & 0.006 & $\begin{array}{c}1.62(1.06- \\
2.58)\end{array}$ & 0.025 & $0.73(0.39-1.48)$ & 0.372 & & \\
\hline
\end{tabular}

Abbreviations: HBs Ag, Hepatitis B surface antigen; HCV Ab, Hepatitis C virus-specific antibody; ICG-R15, Indocyanine green retention rate at 15 minutes; AFP, Alpha-fetoprotein; DCP, Des-gamma-carboxy prothrombin; CA19-9, Carbohydrate antigen 19-9; LN, Lymph node; HR, Hazard ratio; CI, Confidence interval.

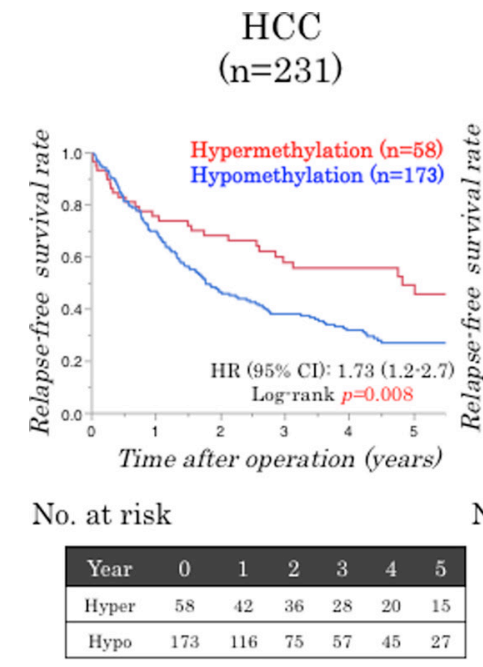

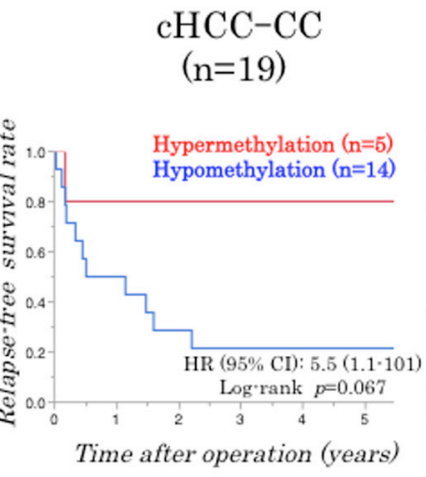

No. at risk

\begin{tabular}{|ccccccc|}
\hline Year & 0 & 1 & 2 & 3 & 4 & 5 \\
\hline Hyper & 5 & 5 & 4 & 4 & 4 & 4 \\
\hline Hypo & 14 & 8 & 5 & 3 & 3 & 3 \\
\hline
\end{tabular}

ICC

$$
(\mathrm{n}=71)
$$

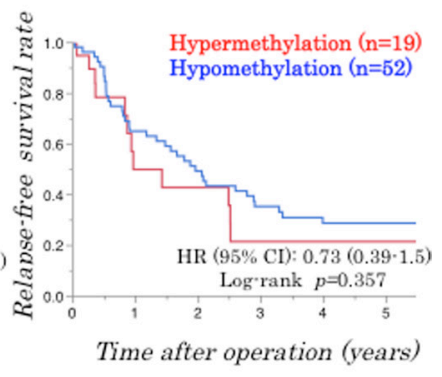

No. at risk

\begin{tabular}{|ccccccc|}
\hline Year & 0 & 1 & 2 & 3 & 4 & 5 \\
\hline Hyper & 19 & 8 & 7 & 4 & 2 & 2 \\
\hline Hyp & 52 & 34 & 26 & 18 & 14 & 12 \\
\hline
\end{tabular}

Figure 3: Relationships between the LINE-1 methylation status and patient survival. LINE-1 hypomethylation is significantly correlated with poor RFS in HCC $(p=0.008)$ but not cHCC-CC $(p=0.067)$ or ICC $(p=0.357)$ patients. 
1 hypermethylation group for cHCC-CC. Therefore, we need additional studies with larger sample sizes to determine whether LINE-1 hypomethylation is indicative of poor prognosis in $\mathrm{CHCC}-\mathrm{CC}$. Third, for ICC, there was no significant difference in LINE-1 methylation levels between the cancerous and noncancerous tissues, and many ICC samples exhibited high LINE-1 methylation levels. Therefore, LINE-1 methylation levels did not influence patient prognoses in ICC. Thus, further larger studies are also required for ICC.

Hepatitis virus infection is a strong prognostic factor of PLC. In the present study, the presence or absence of HCV infection had significant effect on the LINE-1 methylation level of patients with $\mathrm{HCC}$
(Table 1). Nishida et al. reported the effect of hepatitis virus infection on epigenetic status in liver diseases [40]. On the other hand, subgroup analysis showed that the risk of recurrence assessed based on LINE1 hypomethylation was higher in the non-hepatitis virus-infected group than that in the infected group (Figure 4). Therefore, especially in patients without hepatitis virus infection, LINE-1 hypomethylation may be an effective biomarker of HCC recurrence. In the current study, comparison of primary tumor and metastatic lesion of LINE-1 methylation level had not been conducted. As Murata et al. reported in colon cancer [41], it can be expected that primary tumor and metastatic lesion are equivalent LINE-1 methylation in

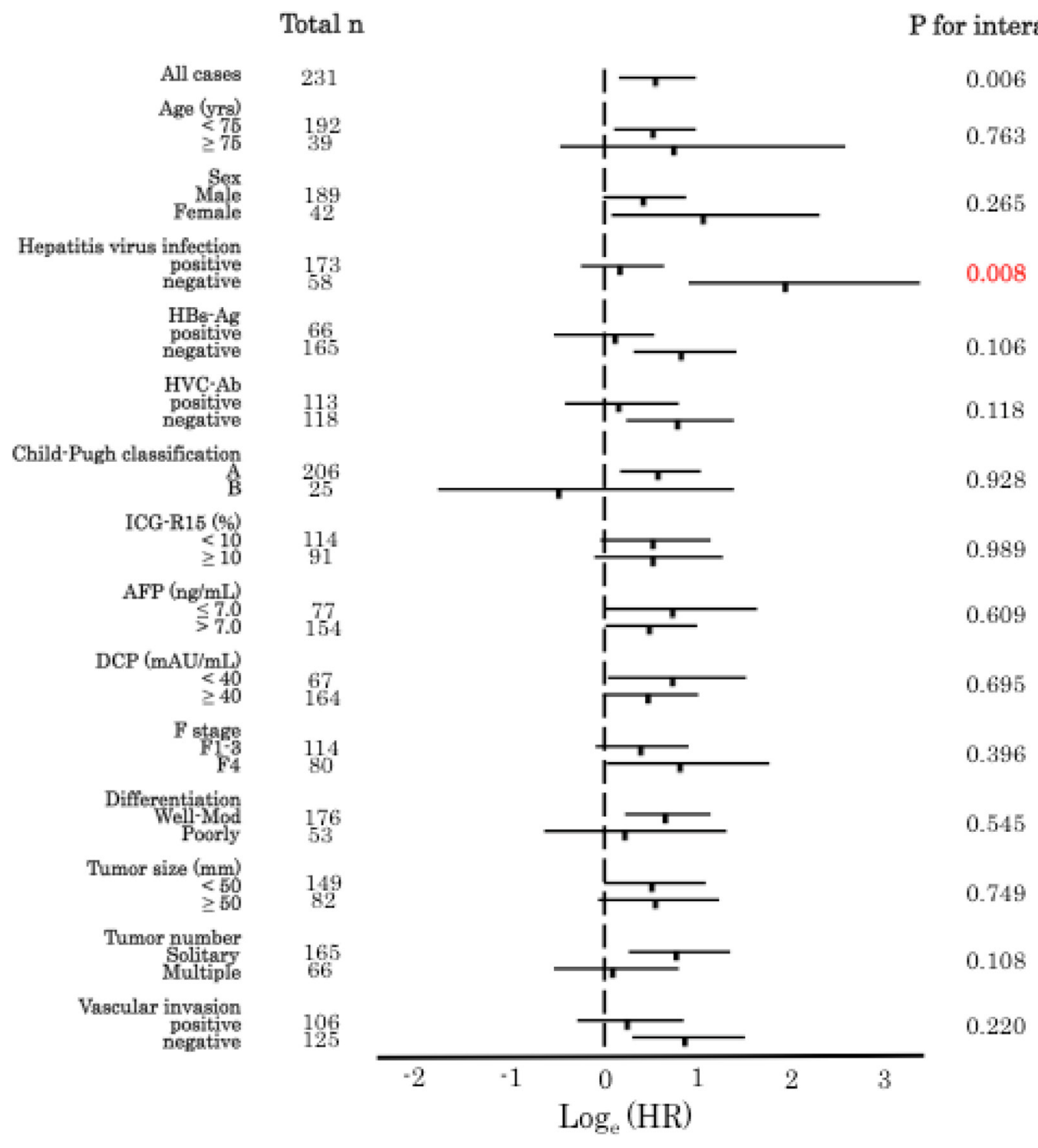

Figure 4: LINE-1 methylation levels in HCC and RFS in various subgroups. $\log _{\mathrm{e}}$ (adjusted HR) plots for RFS rates in the LINE-1 hypomethylation (Q2-4) and hypermethylation (Q1) groups. Values corresponding to 95\% CIs are also indicated. The relationship between LINE-1 methylation level and the RFS rate was significantly modified by hepatitis virus infection $(p$ for interaction $=0.008)$. Bars indicated $95 \%$ confidence intervals. 
HCC. Furthermore, as you know, there are "metastasis" and "multicentric recurrence" in the recurrence pattern of HCC. The methylation level of LINE-1 in cancerous tissue may be useful for this diagnosis, therefore, it may be worthwhile to collect many clinical samples of recurrent resections of HCC.

Table 1 also showed that F4 of noncancerous lesion or higher DCP were significantly associated with lower LINE-1 methylation of HCC. In our own series, there was no statistically difference in the LINE-1 methylation level between F1-3 and F4 of noncancerous lesions (data not shown). There is a possibility that tumor environments such as F4 would affect the LINE-1 methylation level of HCC; however further in vitro study would need to confirm this phenomenon. As for DCP, we could not found any papers which refer the association between the higher DCP and the lower LINE-1 methylation level of HCC. DCP is well known to be a novel biomarker of malignant behavior of HCC; therefore, the association between DCP and LINE-1 methylation would be the important theme to investigate.

There are some limitations in this study, such as the small number of cHCC-CC and ICC cases. The incidence of cHCC-CC and ICC has been reported to vary from fewer than $1 \%$ to approximately $5-10 \%$ of PLC cases. Therefore, a multicenter study with a large number of cHCC-CC or ICC cases, such as a nationwide surveillance study, may be required.

In summary, we evaluated LINE-1 methylation levels using pyrosequencing and examined the prognostic values of LINE-1 hypomethylation in 321 patients with PLC. First, we could confirm prognostic significance of LINE-1 hypomethylation in HCC by using a greater number of cases. Second, LINE-1 hypomethylation was found to be not associated with poor prognosis in ICC and $\mathrm{cHCC}-\mathrm{CC}$ patients. Even in the same primary liver cancer, LINE-1 methylation statuses were different. The mechanisms of epigenetic regulation in PLC and its relationship with cancer initiation and progression should be explored.

\section{MATERIALS AND METHODS}

\section{Study subjects}

$\mathrm{HCC}, \mathrm{cHCC}-\mathrm{CC}$ and ICC samples were collected from patients who had undergone surgical resection as their first therapy at Kumamoto University Hospital (Kumamoto, Japan) between January 2000 and February 2016. Three hundred and twenty-one patients were finally included in the current study. Patients were followed up at 1- to 3-month intervals until death or until July 1, 2017, whichever came first. Relapse-free survival (RFS) was defined as the duration between the operation and the date when cancer recurrence was observed. Overall survival (OS) was defined as the duration between the operation and the date of death. Written informed consent was obtained from each patient, and the study procedures were approved by the institutional review board.

\section{DNA extraction}

Several $10-\mu m$-thick sections of formalin-fixed paraffin-embedded (FFPE) tumor tissues were stained with hematoxylin and eosin (H\&E), and the tumor slides were reviewed by a pathologist who marked the areas corresponding to the tumor and noncancerous liver parenchyma. Cancer tissues without stromal areas were also marked. In each case, as much of the cancer tissues without stroma as possible were macroscopically scraped off from the slides as previously described [17, 21-24, 28, 42]. The section area depended on the size of the tissue (Supplementary Figure 3). Then, DNA was extracted using a QIAamp DNA FFPE Tissue Kit (Qiagen, Valencia, CA, USA).

\section{Sodium bisulfite treatment and pyrosequencing for LINE-1 methylation assessment}

Genomic DNA was treated with sodium bisulfite using an EpiTect Bisulfite Kit (Qiagen) as previously described [17, 21-24, 28, 42]. Polymerase chain reaction (PCR) and subsequent pyrosequencing of LINE-1 were performed as previously described using the PyroMark kit (Qiagen) [17, 21-24, 28, 42]. This assay amplifies a region of LINE-1 containing four CpG sites (base positions 305331 in Accession No. X58075). In each tumor sample, the overall LINE-1 methylation level was determined based on the average relative amount of cytosine $(\mathrm{C})$ residues among the four CpG sites (Supplementary Figure 4). Since several reports have shown that hepatocytes can differentiate into cholangiocytes, which can give rise to $\mathrm{CHCC}-\mathrm{CC}$ and ICC $[7,43,44]$, we considered LINE-1 methylation levels in the liver parenchyma in the calculation of overall LINE-1 methylation levels in noncancerous tissues, as well as in cHCC-CC and ICC tissues.

\section{Statistical analysis}

The clinicopathological characteristics were summarized using descriptive statistics. We compared the means using Student's $t$ test and paired $t$ test and analysis of variance between the two groups. We staged LN status using the seventh edition of the American Joint Committee on Cancer (AJCC)/International Union Against Cancer (UICC) staging manual [45] and F stage using New Inuyama classification [46]. The survival curves were estimated using the Kaplan-Meier method and compared using the log-rank test. Hazard ratios (HRs) and 95\% confidence intervals (CIs) were calculated using Cox proportional hazard models. The prognostic factors for RFS and OS in HCC and ICC patients were assessed using the Cox proportional hazard models with the backward 
elimination method. In addition, to assess interactions among variables, the LINE-1 methylation level was crosscorrelated with another variable of interest via univariate Cox modeling in HCC, and the interaction was evaluated using the Wald test. All $p$-values were two-sided, and values lower than 0.05 were considered statistically significant. JMP (version 12, SAS Institute, Cary, NC, USA) was used for statistical analyses.

\section{Abbreviations}

PLC: Primary liver cancer; HCC: Hepatocellular carcinoma; ICC: Intrahepatic cholangiocarcinoma; cHCC$\mathrm{CC}$ : combined hepatocellular and cholangiocarcinoma; LINE-1: Long interspersed element-1; HBV: hepatitis B virus; $\mathrm{HCV}$ : hepatitis $\mathrm{C}$ virus; NAFLD: non-alcoholic fatty liver disease; RFS: Relapse-free survival; OS: Overall survival; FFPE: formalin-fixed paraffin-embedded; H\&E: hematoxylin and eosin; SD: Standard deviation; DCP: Des-gamma-carboxy prothrombin; IDH: isocitrate dehydrogenase; TET: Ten-eleven translocation.

\section{Author contributions}

Conception and design: T. Miyata, Y. Yamashita, Y. Baba. K. Harada. Development of methodology: T. Miyata, Y. Yamashita. Acquisition of data (provided animals, acquired and managed patients, provided facilities, etc.): T. Miyata, T. Yamao, N. Umezaki, M. Tsukamoto, Y. Kitano, K. Yamamura, K. Arima; Analysis and interpretation of data (e.g., statistical analysis, biostatistics, computational analysis): T. Miyata, M. Shimokawa; Writing, review, and/or revision of the manuscript: T. Miyata, Y. Yamashita, Y. Baba, H. Baba; Administrative, technical, or material support (i.e., reporting or organizing data, constructing databases): T. Miyata, Y. Yamashita, Y. Baba, M. Shimokawa, S. Nakagawa, H. Baba; Study supervision: Y. Yamashita, K. Imai, D. Hashimoto, A. Chikamoto, H. Baba.

\section{ACKNOWLEDGMENTS}

We thank Keisuke Miyake and Yoko Ogata for assisting with experimental techniques. We greatly appreciate their help.

\section{CONFLICTS OF INTEREST}

None.

\section{FUNDING}

This work was not funded.

\section{REFERENCES}

1. Torre LA, Bray F, Siegel RL, Ferlay J, Lortet-Tieulent J, Jemal A. Global cancer statistics, 2012. CA Cancer J Clin. 2015; 65:87-108.

2. Blechacz B, Gores GJ. Cholangiocarcinoma: advances in pathogenesis, diagnosis, and treatment. Hepatology. 2008; 48:308-321.

3. Sia D, Tovar V, Moeini A, Llovet JM. Intrahepatic cholangiocarcinoma: pathogenesis and rationale for molecular therapies. Oncogene. 2013; 32:4861-4870.

4. Shin HR, Oh JK, Masuyer E, Curado MP, Bouvard V, Fang YY, Wiangnon S, Sripa B, Hong ST. Epidemiology of cholangiocarcinoma: an update focusing on risk factors. Cancer Sci. 2010; 101:579-585.

5. El-Serag HB, Rudolph KL. Hepatocellular carcinoma: epidemiology and molecular carcinogenesis. Gastroenterology. 2007; 132:2557-2576.

6. Baffy G, Brunt EM, Caldwell SH. Hepatocellular carcinoma in non-alcoholic fatty liver disease: an emerging menace. J Hepatol. 2012; 56:1384-1391.

7. Oikawa T. Cancer Stem cells and their cellular origins in primary liver and biliary tract cancers. Hepatology. 2016; 64:645-651.

8. Tyson GL, El-Serag HB. Risk factors for cholangiocarcinoma. Hepatology. 2011; 54:173-184.

9. Theise ND, Park Y, Nakanuma Y. Combined hepatocellularcholangiocarcinoma. In: Bosman FT, Carneiro F, Hruban RH, Theise ND, eds. WHO Classification of Tumours of the Digestive System. 4th ed. Lyon, France IARC. 2010; 225-227.

10. Baylin SB, Esteller M, Rountree MR, Bachman KE, Schuebel K, Herman JG. Aberrant patterns of DNA methylation, chromatin formation and gene expression in cancer. Hum Mol Genet. 2001; 10:687-692.

11. Issa JP. CpG island methylator phenotype in cancer. Nat Rev Cancer. 2004; 4:988-993.

12. Esteller M. The necessity of a human epigenome project. Carcinogenesis. 2006; 27:1121-1125.

13. Jones PA, Baylin SB. The epigenomics of cancer. Cell. 2007; 128:683-692.

14. Rodriguez-Paredes M, Esteller M. Cancer epigenetics reaches mainstream oncology. Nat Med. 2011; 17:330-339.

15. Gaudet F, Hodgson JG, Eden A, Jackson-Grusby L, Dausman J, Gray JW, Leonhardt H, Jaenisch R. Induction of tumors in mice by genomic hypomethylation. Science. 2003; 300:489-492.

16. Suzuki K, Suzuki I, Leodolter A, Alonso S, Horiuchi S, Yamashita K, Perucho M. Global DNA demethylation in gastrointestinal cancer is age dependent and precedes genomic damage. Cancer Cell. 2006; 9:199-207.

17. Baba Y, Huttenhower C, Nosho K, Tanaka N, Shima K, Hazra A, Schernhammer ES, Hunter DJ, Giovannucci EL, 
Fuchs CS, Ogino S. Epigenomic diversity of colorectal cancer indicated by LINE-1 methylation in a database of 869 tumors. Mol Cancer. 2010; 9:125.

18. Eden A, Gaudet F, Waghmare A, Jaenisch R. Chromosomal instability and tumors promoted by DNA hypomethylation. Science. 2003; 300:455.

19. Cordaux R, Batzer MA. The impact of retrotransposons on human genome evolution. Nat Rev Genet. 2009; 10:691-703.

20. van Hoesel AQ, van de Velde CJ, Kuppen PJ, Liefers GJ, Putter H, Sato Y, Elashoff DA, Turner RR, Shamonki JM, de Kruijf EM, van Nes JG, Giuliano AE, Hoon DS. Hypomethylation of LINE-1 in primary tumor has poor prognosis in young breast cancer patients: a retrospective cohort study. Breast Cancer Res Treat. 2012; 134:1103-1114.

21. Iwagami S, Baba Y, Watanabe M, Shigaki H, Miyake $\mathrm{K}$, Ishimoto $\mathrm{T}$, Iwatsuki M, Sakamaki K, Ohashi Y, Baba H. LINE-1 hypomethylation is associated with a poor prognosis among patients with curatively resected esophageal squamous cell carcinoma. Ann Surg. 2013; 257:449-455.

22. Ogino S, Nosho K, Kirkner GJ, Kawasaki T, Chan AT, Schernhammer ES, Giovannucci EL, Fuchs CS. A cohort study of tumoral LINE-1 hypomethylation and prognosis in colon cancer. J Natl Cancer Inst. 2008; 100:1734-1738.

23. Shigaki H, Baba Y, Watanabe M, Murata A, Iwagami S, Miyake K, Ishimoto T, Iwatsuki M, Baba H. LINE-1 hypomethylation in gastric cancer, detected by bisulfite pyrosequencing, is associated with poor prognosis. Gastric Cancer. 2013; 16:480-487.

24. Yamamura K, Kosumi K, Baba Y, Harada K, Gao F, Zhang X, Zhou L, Kitano Y, Arima K, Kaida T, Takeyama H, Higashi T, Imai K, et al. LINE-1 methylation level and prognosis in pancreas cancer: pyrosequencing technology and literature review. Surg Today 2017; 47:1450-1459.

25. Yang AS, Estecio MR, Doshi K, Kondo Y, Tajara EH, Issa JP. A simple method for estimating global DNA methylation using bisulfite PCR of repetitive DNA elements. Nucleic Acids Res. 2004; 32:e38.

26. Ogino S, Kawasaki T, Nosho K, Ohnishi M, Suemoto Y, Kirkner GJ, Fuchs CS. LINE-1 hypomethylation is inversely associated with microsatellite instability and $\mathrm{CpG}$ island methylator phenotype in colorectal cancer. Int $\mathrm{J}$ Cancer. 2008; 122:2767-2773.

27. Irahara N, Nosho K, Baba Y, Shima K, Lindeman NI, Hazra A, Schernhammer ES, Hunter DJ, Fuchs CS, Ogino S. Precision of pyrosequencing assay to measure LINE-1 methylation in colon cancer, normal colonic mucosa, and peripheral blood cells. J Mol Diagn. 2010; 12:177-183.

28. Harada K, Baba Y, Ishimoto T, Chikamoto A, Kosumi K, Hayashi H, Nitta H, Hashimoto D, Beppu T, Baba H. LINE-1 methylation level and patient prognosis in a database of 208 hepatocellular carcinomas. Ann Surg Oncol. 2015; 22:1280-1287.

29. Udali S, Guarini P, Moruzzi S, Ruzzenente A, Tammen SA, Guglielmi A, Conci S, Pattini P, Olivieri O, Corrocher R, Choi SW, Friso S. Global DNA methylation and hydroxymethylation differ in hepatocellular carcinoma and cholangiocarcinoma and relate to survival rate. Hepatology. 2015; 62:496-504.

30. Nakamura H, Arai Y, Totoki Y, Shirota T, Elzawahry A, Kato M, Hama N, Hosoda F, Urushidate T, Ohashi S, Hiraoka N, Ojima H, Shimada K, et al. Genomic spectra of biliary tract cancer. Nat Genet. 2015; 47:1003-1010.

31. Wang P, Dong Q, Zhang C, Kuan PF, Liu Y, Jeck WR, Andersen JB, Jiang W, Savich GL, Tan TX, Auman JT, Hoskins JM, Misher AD, et al. Mutations in isocitrate dehydrogenase 1 and 2 occur frequently in intrahepatic cholangiocarcinomas and share hypermethylation targets with glioblastomas. Oncogene. 2013; 32:3091-3100.

32. Borger DR, Tanabe KK, Fan KC, Lopez HU, Fantin VR, Straley KS, Schenkein DP, Hezel AF, Ancukiewicz M, Liebman HM, Kwak EL, Clark JW, Ryan DP, et al. Frequent mutation of isocitrate dehydrogenase (IDH) 1 and IDH2 in cholangiocarcinoma identified through broad-based tumor genotyping. Oncologist. 2012; 17:72-79.

33. Clark O, Yen K, Mellinghoff IK. Molecular Pathways: Isocitrate Dehydrogenase Mutations in Cancer. Clin Cancer Res. 2016; 22:1837-1842.

34. Murata A, Baba Y, Ishimoto T, Miyake K, Kosumi K, Harada K, Kurashige J, Iwagami S, Sakamoto Y, Miyamoto Y, Yoshida N, Yamamoto M, Oda S, et al. TET family proteins and 5-hydroxymethylcytosine in esophageal squamous cell carcinoma. Oncotarget. 2015; 6:23372-23382. https://doi. org/10.18632/oncotarget.4281.

35. Berdasco M, Esteller M. Aberrant epigenetic landscape in cancer: how cellular identity goes awry. Dev Cell. 2010; 19:698-711.

36. Weber B, Kimhi S, Howard G, Eden A, Lyko F. Demethylation of a LINE-1 antisense promoter in the cMet locus impairs Met signalling through induction of illegitimate transcription. Oncogene. 2010; 29:5775-5784.

37. Wu H, Tao J, Li X, Zhang T, Zhao L, Wang Y, Zhang L, Xiong J, Zeng Z, Zhan N, Steer CJ, Che L, Dong M, et al. MicroRNA-206 Prevents the Pathogenesis of Hepatocellular Carcinoma Via Modulating Expression of cMet and Cdk6. Hepatology 2017; 66:1952-1967.

38. Poon RT, Fan ST, Lo CM, Liu CL, Wong J. Intrahepatic recurrence after curative resection of hepatocellular carcinoma: long-term results of treatment and prognostic factors. Ann Surg. 1999; 229:216-222.

39. Chen WT, Chau GY, Lui WY, Tsay SH, King KL, Loong $\mathrm{CC}, \mathrm{Wu} \mathrm{CW}$. Recurrent hepatocellular carcinoma after hepatic resection: prognostic factors and long-term outcome. Eur J Surg Oncol. 2004; 30:414-420. 
40. Nishida N, Iwanishi M, Minami T, Chishina H, Arizumi T, Takita M, Kitai S, Yada N, Ida H, Hagiwara S, Minami Y, Ueshima K, Sakurai T, et al. Hepatic DNA Methylation Is Affected by Hepatocellular Carcinoma Risk in Patients with and without Hepatitis Virus. Dig Dis. 2015; 33:745-750.

41. Murata A, Baba Y, Watanabe M, Shigaki H, Miyake K, Ishimoto T, Iwatsuki M, Iwagami S, Sakamoto Y, Miyamoto Y, Yoshida N, Nosho K, Baba H. Methylation levels of LINE-1 in primary lesion and matched metastatic lesions of colorectal cancer. Br J Cancer. 2013; 109:408-415.

42. Iwagami S, Baba Y, Watanabe M, Shigaki H, Miyake K, Ida S, Nagai Y, Ishimoto T, Iwatsuki M, Sakamoto Y, Miyamoto Y, Baba H. Pyrosequencing assay to measure LINE-1 methylation level in esophageal squamous cell carcinoma. Ann Surg Oncol. 2012; 19:2726-2732.

43. Sekiya S, Suzuki A. Intrahepatic cholangiocarcinoma can arise from Notch-mediated conversion of hepatocytes. J Clin Invest. 2012; 122:3914-3918.
44. Fan B, Malato Y, Calvisi DF, Naqvi S, Razumilava N, Ribback S, Gores GJ, Dombrowski F, Evert M, Chen X, Willenbring H. Cholangiocarcinomas can originate from hepatocytes in mice. J Clin Invest. 2012; 122:2911-2915.

45. Edge S, Byrd DR, Compton CC, Fritz AG, Greene FL, Trotti A. AJCC Cancer Staging Manual (7th ed). New York, NY, Springer. 2010.

46. Ichida F, Tsuji T, Omata M, Ichida T, Inoue K, Kamimura T, Yamada G, Hino K, Yokosuka O, Suzuki H. New Inuyama classification; new criteria for histological assessment of chronic hepatitis. International Hepatology Communications. 1996; 6:112-119. 